\title{
A micromachined interface for airborne sample-to-liquid transfer and its application in a biosensor system $\dagger$
}

\author{
Thomas Frisk, ${ }^{* a}$ David Rönnholm, ${ }^{b}$ Wouter van der Wijngaart ${ }^{a}$ and Göran Stemme ${ }^{a}$ \\ Received 22nd May 2006, Accepted 30th August 2006 \\ First published as an Advance Article on the web 20th September 2006 \\ DOI: $10.1039 / \mathrm{b} 612526 n$
}

A novel micromachined interface for airborne sample-to-liquid adsorption and droplet-to-liquid transfer was designed and fabricated. It enables a robust sheet liquid flow serving as an adsorption site. The interface was characterised for flow and pressure properties and tested successfully for the transfer/adsorption of different samples. A qualitative theoretical model of the device characteristics is presented. We also used the interface to introduce a novel method and system for fast detection of dust- and vapour-based narcotics and explosives traces. The microfluidic vapourto-liquid adsorption interface was coupled to a set of downstream QCM sensors. The system was tested successfully, with $50 \mathrm{ng}$ cocaine samples rendering $15 \mathrm{~Hz}$ frequency shifts and with $100 \mathrm{ng}$ heroine samples rendering $50 \mathrm{~Hz}$ frequency shifts. Gravitation invariance of the open liquid interface was demonstrated successfully, with the interface mounted upside down as well as vertically. The detection time was reduced to half of the time needed in previous systems. Machine size, weight and cost were reduced.

\section{Introduction}

Detection, monitoring, and public surveillance of chemicals e.g. explosives, drugs and dioxins as well as other pollutants and contaminants, and of biological substances e.g. bacteria or fungi is an important part of security, environmental, industrial and military activities in today's world. Sample-toliquid interfacing is required in all analysis systems where chemical analysis of dust particles, vapour based samples, and aerosols is performed in a liquid environment. Analysis of liquid aggregates is by far the most common analysis form for chemicals, but the substance of interest is often airborne. The device presented here provides an open liquid-air interface for direct vapour-to-liquid, as well as liquid-to-liquid, sample capture and transfer. Interfacing droplets to liquid was shown previously ${ }^{1,2}$ though, and will not be discussed further.

Component design requirements include:

1. Interface efficiency demands a large interface area $\left(\sim \mathrm{cm}^{2}\right)$ as an adsorption/condensation site, and a small total liquid volume to obtain as high a sample concentration as possible.

2. Appropriate pressure-flow $(P-Q)$ characteristics i.e. allowing sufficient liquid flow $\left(\sim \mu \mathrm{l} \mathrm{s} \mathrm{s}^{-1}\right)$ with low flow resistance $(\sim \mathrm{kPa})$.

3. Easy priming of liquid through the devices, e.g. by capillary filling.

4. Prevention of device flooding-robustness issue.

5. Prevention of device drying or downstream air bubble introduction-robustness issue.

${ }^{a}$ Microsystem Technology Laboratory, School of Electrical Engineering, Royal Institute of Technology, SE-100 44 Stockholm, Sweden.

E-mail:Thomas.Frisk@ee.kth.se; Fax: +46-8-1008 58;

Tel: +46-70-5950842

${ }^{b}$ Biosensor Applications Sweden AB, Ursviksvägen 131A, SE-

17446 Sundbyberg, Sweden

$\uparrow$ The HTML version of this article has been enhanced with colour images.
6. Minimisation of temperature sensitivity, i.e. prevention of liquid concentration changes due to evaporation.

7. Uncomplicated design for ease of fabrication and usage.

For practical use, considerations to take into account include:

8. Gravitational invariance.

9. Easy cleaning and/or regeneration of the device.

In particular, there exists a need for fast, portable, yet sensitive electronic noses for narcotics and explosives detection, e.g. in customs, prisons and transportation industry. Commercially available quartz crystal microbalance (QCM) based detectors ${ }^{3}$ require the sample to be dissolved in a liquid environment at the sensing element closed to ambient conditions. Heavy, bulky and costly system components are used to (1) transfer sample from dust collected on a paper filter to the on-system liquid environment and (2) periodically renew selective antibodies at the sensor surface. The commercially available BIOSENS ${ }^{(i x)} 3$ machine uses robotics, liquid looping, three pumps, and three valves ${ }^{46}$ and is therefore not portable; portability requires light, small systems in which the orientation of the device does not affect its performance (e.g. the behaviours of fluids are not affected by gravitational forces). Other requirements include a low "dead" internal surface to reduce parasitic binding sites for sample and antibodies and a low dead volume to reduce sample dispersion and increase measurement times.

This paper describes both a novel interface device and its integration for sample-to-liquid usage. The open "wet" micromachined surface over which a water phosphate buffer solution (PBS) flows, was integrated and tested with a commercial QCM based system $\left(\right.$ BIOSENS $\left.^{(\mathbb{1}}\right)$, addressing all of the above requirements.

\section{Device description}

The interface component consists of a micromachined surface over which a thin sheet of constant liquid flow is guided in 
contact with the surrounding atmosphere (Fig. 1). The device surface consists of a regular distribution of cylindrical pillars in a checker board pattern.

Liquid is drawn by capillary action between the pillars on the chip surface, with surface tension preventing liquid wetting the pillar tops. The pillar height $h$ thus determines the liquid sheet thickness.

Liquid is added and removed from the top of the chip surface via hydrophobic fluid connectors with stainless steel tube interfaces. The connectors feature a manifold design for even and efficient filling and draining of the interface component.

The liquid-air interface forms a condensation site for vapour or aerosol; particles may also be trapped on the liquid surface. Liquid drops dispensed onto the liquid-air surface will also merge with the free flowing liquid sheet. The flow in the liquid sheet carries sample to the outlet of the device and can be collected at the device outlet for downstream analysis or storage.

In its normal mode of operation, liquid fills the device up to the full pillar height.

Two failure modes exist: drying of the interface, leading to downstream air bubble introduction; and flooding of the interface, resulting in overflow effects. ${ }^{2}$

The capillary forces on the liquid between the pillars counteract drying and air bubbles entering the interface outlet, whereas surface tension provides overflow prevention.
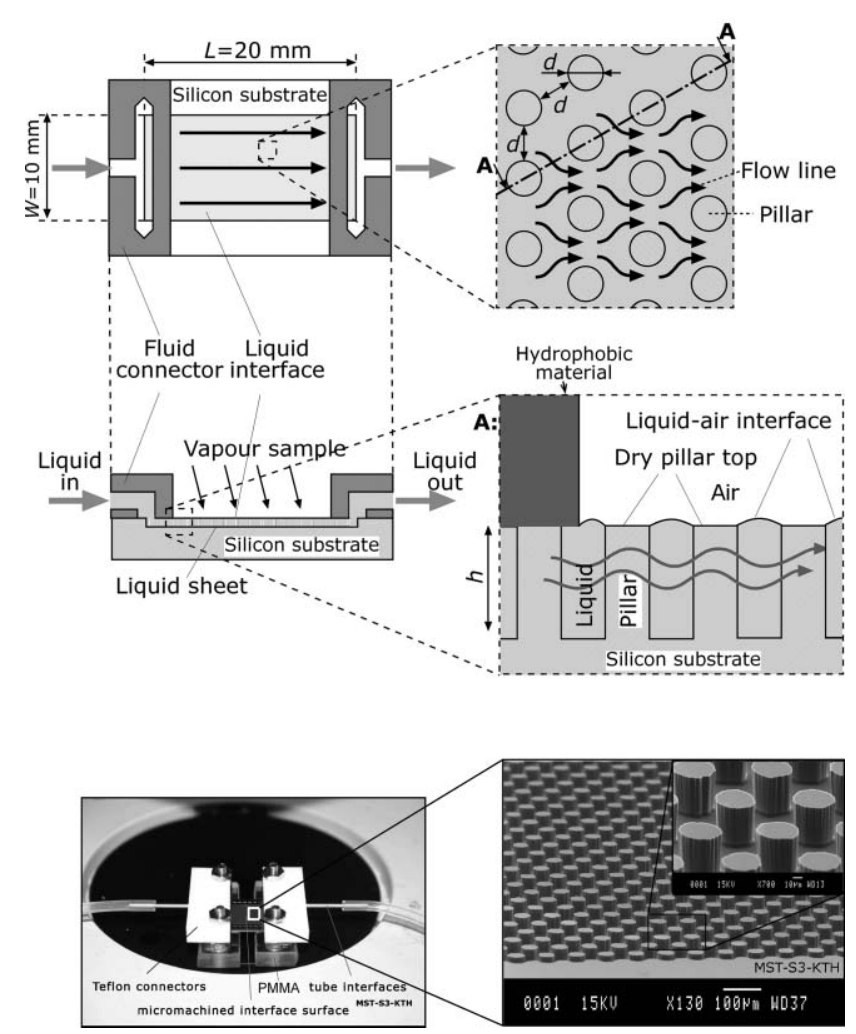

Fig. 1 Schematic top view (top) and cross-sectional view (middle) of the air-liquid interface component. The left bottom photograph shows a $2 \times 1 \mathrm{~cm}^{2}$ interface chip with Teflon ${ }^{(i x}$ connectors and stainless steel tube interfaces with silicone tubing. The SEM image shows a close-up of the microstructured silicon surface with $50 \mu \mathrm{m}$ tall pillar structures.
Overflow of the liquid on top of the pillars can be actively controlled by adjusting the supply pressure or drain pressure. This can be beneficial for rinsing and cleaning contaminants from the surface. An intentional overflow with subsequent flooding produced by raising inlet and outlet pressures will cause high flow rates of fluid and a cleaning effect.

To prevent unintentional flooding at the connector-chip-air contact line, hydrophobic connector materials, such as Teflon $^{(i x)}$ or PDMS are preferred for the fluid connectors.

\section{Fabrication}

The device consists of a silicon chip, plastic inlet and outlet manifolds with tubing, and a plastic or metallic support clamped together with M2.5 screws (Fig. 1 and 4).

For the characterisation of the interface component, a Teflon ${ }^{(i)}$ inlet and outlet manifold with stainless steel tubing was used. For integration trials/evaluation of the component, a PEEK $^{(i i}$ ring with drilled inlet and outlet manifold channels was designed, and PEEK ${ }^{(i i}$ tubing glued to it.

The silicon surface microstructuring was performed with standard methods including a single photolithography, deep reactive ion etching (DRIE), oxidation and final dicing of the 4 inch, $525 \mu \mathrm{m}$ thick, Si wafers. The wet oxidation of the substrates in a furnace oven $\left(1100{ }^{\circ} \mathrm{C}\right)$ formed a $1 \mu \mathrm{m}$ thick layer of $\mathrm{SiO}_{2}$ on the surface and removed any residual material from the etch process.

The following geometries were tested in device characterisation: interface area width $W=1 \mathrm{~cm}$, length $L=2 \mathrm{~cm}$, pillar diameter $d=40 \mu \mathrm{m}$, heights $h=50$ and $h=100 \mu \mathrm{m}$ and interpillar distance $d$ provided a $\sim 2 \mathrm{~cm}^{2}$ interface area containing 10 and $20 \mu \mathrm{l}$ liquid volumes respectively. In device evaluation for drug detection the effective interface diameter was $10 \mathrm{~mm}$ defined by the manifold ring. Pillar height $h=100 \mu \mathrm{m}$, other dimensions kept same.

\section{Theoretical model}

The flow behaviour of liquid on a regularly microstructured open surface is modelled as a 2-D extension of flow through a single open microcanal. ${ }^{2}$

For theoretical analysis we model the device surface as $n=$ $W / 2 d$ parallel microcanals of width $d$ and height $h$ with straight sidewalls. We assume parallel plate Poiseuille flow (i.e. stationary, laminar flow of an incompressible Newtonian liquid with viscosity $\mu$ ) between the sidewalls and we neglect the channel bottom.

The viscous flow resistance, ${ }^{7}$ i.e. pressure drop $\Delta P_{1}=P_{\text {in }}-$ $P_{\text {out }}$ per flow $Q$, for such geometry can be expressed as:

$$
\frac{\Delta P_{1}}{Q}=k \frac{1}{W / d} \frac{L \mu}{h d^{3}}
$$

with $k$ being the flow resistance coefficient of this model. For the parallel microcanal model one can deduce the value $k=48$.

Note that the internal liquid pressure $P_{1}(x)$, with $x$ being the position along the flow direction, drops along the length $L$ of the liquid interface (canal) due to viscous flow losses, and that the liquid pressure at the upstream side of the device is always larger than at the downstream side. 


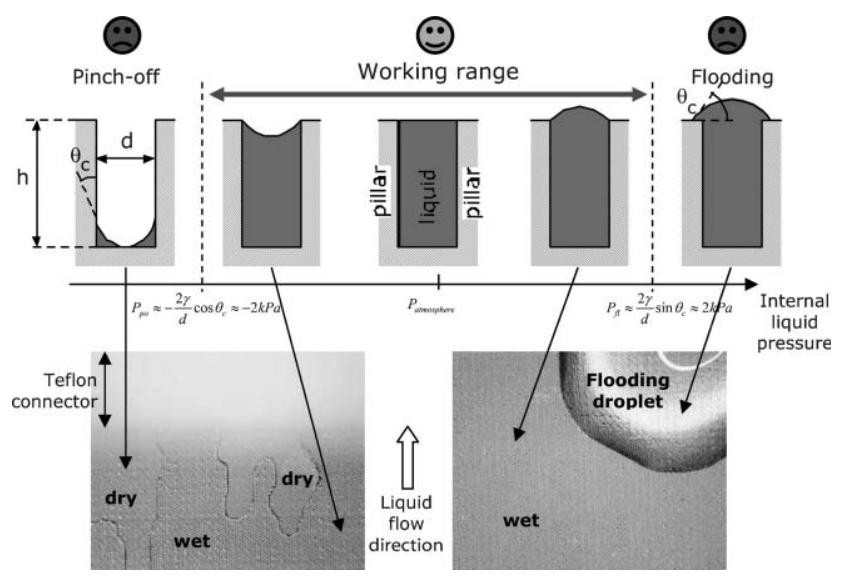

Fig. 2 Schematic cross-sectional view of the device wetting and the liquid-air interface geometry in the device at different internal liquid pressures $P_{1}$ (top) and top view microscope photographs illustrating the different states (bottom).

The two device failure mechanisms are shown in Fig. 2. The pressure drop $\Delta P$ over a stationary curved fluid interface is expressed with the Young-Laplace equation:

$$
\Delta P=\gamma\left[\frac{1}{R_{1}}+\frac{1}{R_{2}}\right]
$$

with $R_{1}$ being the radius in the $y z$-plane, $R_{2}$ being the radius in the $x z$-plane, and $\gamma$ being the surface tension of the liquid.

Assuming a cylindrical liquid-gas interface $\left(R_{1}=R ; R_{2}=\right.$ $\infty$ ), one can deduce ${ }^{2}$ that device flooding (i.e. liquid covering the pillar tops) occurs where the internal liquid pressure

$$
P_{1}>P_{\mathrm{fl}}=\frac{2 \gamma}{d} \sin \theta_{\mathrm{c}}
$$

with $\theta_{\mathrm{c}}$ being the contact angle between the liquid and the solid canal material.

Device pinch-off (i.e. drying) and downstream air bubble introduction occur when the liquid pressure $P_{1}$ is below the capillary suction of the device:

$$
P_{1}<P_{\mathrm{po}}=-\frac{2 \gamma}{d} \cos \theta_{c}
$$

There thus exists a "safe" liquid pressure range — a working range: $P_{\mathrm{f} 1}>P_{1}(x)>P_{\mathrm{po}}$.

The spacing $d$ between the pillars forms a critical design parameter, and its choice is a trade-off.

On one hand, eqn (1) shows that the viscous pressure drop increases quadratically with decreasing inter-pillar spacing $d$. Depending on whether the flow is controlled with a pressure source (e.g. hydrostatically) or with a flow source (e.g. syringe pump), and depending on whether the flow control is upstream or downstream of the device, a large pressure drop $\Delta P$ over the device can lead to either flooding on the upstream side, or pinch-off at the downstream side.

On the other hand, eqn (3) and (4) show that decreasing inter-pillar spacing decreases the pinch-off pressure and increases the overflow robustness. However, these effects scale only linearly with $d$.
The choice of pillar height $h$ is also a trade-off. On one hand, small values of $h$ increase the interface area per liquid volume, and thus the sample concentration in the adsorbing liquid. The decreased flow cross-sectional area, on the other hand, increases the viscous pressure drop over the device, and thus the risk for flooding or pinch-off. For the device to be gravitationally insensitive, length $L$ and width $W$ should be kept small enough so that the hydrostatic pressure change occurring in the device when it is positioned vertically does not influence the pressure working range of the device. For example, a device with $L=2 \mathrm{~cm}$ repositioned vertically, will cause a hydrostatic pressure change of $\rho g L=196 \mathrm{~Pa}$ and will not lead to pinch-off or flooding in the working-range of the device presented here.

Another design constraint is that, with changing liquid consistency or temperature during measurement, the surface tension alters. However, these effects or problems related to them have not been observed during our experiments.

\section{Experimental—characterisation setup and results}

The pressure-flow characteristics of the device were measured with DI water. The setup with hydrostatic upstream and downstream pressure control is shown in Fig. 3. The device was positioned at a fixed height. Inlet and outlet height were controlled with table lab jacks, while the mass flow was measured with an electronic scale at the outlet beaker. In this setup, the measurement results for devices with different geometries under different working conditions are shown. The top graph shows the measured liquid volume flow $Q$ versus the applied pressure difference $P_{\text {in }}-P_{\text {out }}$ between the device inlet and outlet. The bottom graph shows the data after normalisation with respect to the pinch-off pressure $P_{\mathrm{po}}$, in which the normalised pressure

$$
P_{\mathrm{N}}=\frac{P_{\text {in }}-P_{\text {out }}}{P_{\text {in }}-P_{\text {po }}}
$$

and the normalised flow

$$
Q_{\mathrm{N}}=\frac{Q}{\frac{L \mu}{W h d^{2}}\left(P_{\mathrm{in}}-P_{\mathrm{po}}\right)} .
$$

Here, the pinch-off value $P_{\text {po }}$ was calculated as

$$
P_{\mathrm{po}}=-a \frac{2 \gamma}{d} \cos \theta_{\mathrm{c}}
$$

with $\gamma=0.073 \mathrm{~N} \mathrm{~m}^{-1}$ and $\cos \left(\theta_{\mathrm{c}}\right)=1$. The factor $a$ was added to account for deviations between the actual and theoretical values of surface tension and contact angle, as well as to account for the geometrical mismatch between the 2-D model and the actual 3-D liquid-air surface geometry. A good match between theory and model was obtained for $a=0.35$.

For the device with spacing $d=20 \mu \mathrm{m}$ and pillar height $h=50 \mu \mathrm{m}$ we measured flows from $0.1 \mu \mathrm{s} \mathrm{s}^{-1}$ to $0.5 \mu 1 \mathrm{~s}^{-1}$. This is the linear flow range of this device. For the device with $d=40 \mu \mathrm{m}$ and $h=100 \mu \mathrm{m}$, the linear operation range was found to be between $1.0 \mu \mathrm{l} \mathrm{s}^{-1}$ and $2.2 \mu \mathrm{l} \mathrm{s}^{-1}$. Pinch-off and overflow regimes were observed in accordance with theory. In correspondence to ${ }^{2}$ previously reported properties of a 

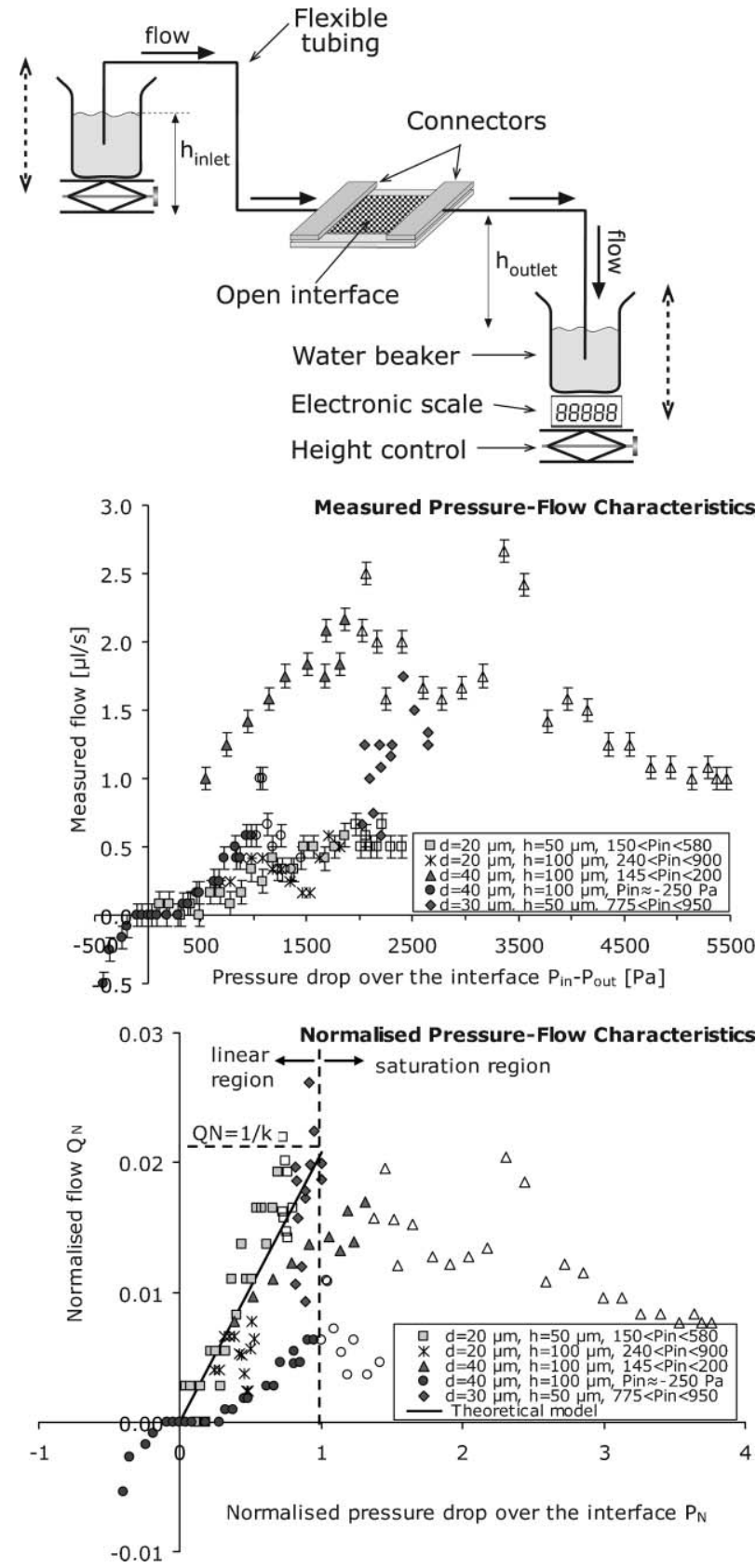

Fig. 3 Characterisation of the fluidic properties of the sample interface. The top illustration shows a schematic of the experimental setup for mass flow measurement with hydrostatic inlet and outlet pressure control. The middle and bottom graphs show pressure-flow measurement data from devices with different values of pillar interdistance $d$ and height $h$ and measured under various inlet pressure bias conditions. The top graph shows the measured volume flow values versus the pressure drop $P_{\text {in }}-P_{\text {out }}$. The error bars indicate the flow measurement accuracy. The bottom graph shows the same data in normalised format $\left(Q_{\mathrm{N}}\right.$ versus $\left.P_{\mathrm{N}}\right)$ illustrating the qualitative match between the measurements and theory. Where $P_{\mathrm{N}}$ values are below unity, the model predicts a linear $P_{\mathrm{N}}-Q_{\mathrm{N}}$ relation with slope $1 / k \approx$ 0.021 . Where $P_{\mathrm{N}}$ values exceed unity, theory predicts that the interface becomes pinched-off at the outlet and flow saturation should occur. Each measurement series obtained with a specific interface component under similar inlet pressure bias is indicated with the same dot shape. Where pinch-off was visually observed, the dots are left unfilled. Elsewhere, the dots are filled. micro-canal device, linear $P-Q$ characteristics were observed at moderate pressure drops, and saturation of flow at large pressure drops where pinch-off occurs.

The system was also tested successfully for liquid droplet interfacing. Droplets of dye were dropped on the interface surface, and the migration of the coloured liquid was observed with the naked eye. The dye residence time in the liquid sheet varied typically between $3 \mathrm{~s}$ and $10 \mathrm{~s}$ depending on the flow rate.

The insensitivity of the device to gravity was tested by varying the position of the device. We tested the device turned upside down as well as tilted and tilted-turned With the upside down device we observed no difference in functionality. With the device vertical we found only a slight performance difference compared to the device in the horizontal position. We detected no difference in performance when the device was operated in a sideways tilted position.

\section{Experimental-integration and system setup}

Finally, liquid sample and vapour sample interfacing was performed successfully.

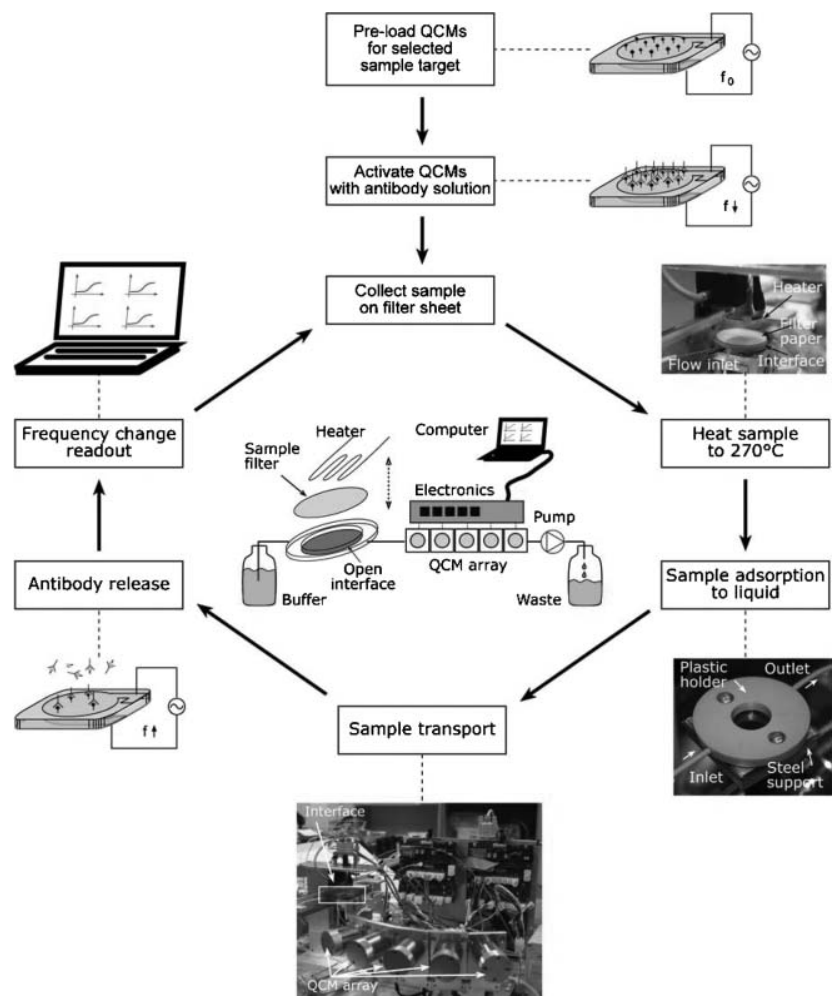

Fig. 4 Schematic of the narcotics detection setup and procedure. The system is illustrated in the centre and consists of a series coupling of interface, detection cells and pump. The measurement procedure includes: (1) the QCM is pre-coated with specific antigens; (2) antibodies are dropped on the interface and bind to the antigens on the surface, after which the resonance frequency drops and stabilises at approx. $10 \mathrm{MHz}$; (3) narcotic sample is collected on a filter sheet; (4) the paper is heated above the interface, whereupon narcotic traces become airborne and adsorb on the liquid interface; (5) target substance molecules enter the QCM sensor cell whereupon antibodies release, causing a weight loss that results in the detection of a drop in resonance frequency. 
The complete measurement system consists of a fluidic serial coupling for running assays in serial (see centre illustration in Fig. 4); a phosphate buffer solution (PBS) reservoir bottle, a peristaltic constant flow pump (100 $\mu 1 \mathrm{~min}^{-1}$ constant flow) and the microfluidic interface component, two heroin sensitive and two cocaine sensitive QCM sensors, ${ }^{3}$ a reference QCM sensor, a peristaltic suction pump and a waste reservoir.

The paper filter is the front-end collector of drug/explosives molecules and particles, and is mounted in a hand-held custom-built collector device for field usage during sample collection. During analysis, the paper is positioned close to the air-liquid interface component. Above it, a controllable heater/oven is mounted to facilitate enough heat for evaporation of substances of interest.

The system further comprises driving and control electronics to accomplish stable frequency measurements of the QCMs in a liquid environment (built by Biosensor Applications, Sweden) and a controlling and logging computer with LabView software (National Instruments, USA).

Five QCMs in series are used, mounted in PEEK ${ }^{(i x)}$ plastic holders accommodating liquid inlet and outlet, electrical interfacing and electromagnetic shielding (the BioCell ${ }^{(i \pi}$ ). Four different surface modifications of the QCM crystals are possible for four different narcotics (or explosives), with the last one being a non-modified reference cell. The QCMs are driven by five custom-made oscillator circuits designed for operation of the quartz crystal in a liquid environment; the base frequency is $10 \mathrm{MHz}$. The loss of mass, through loss of material (antibodies), attached to the surface of the crystal during a 'hit', will cause a resonance frequency shift upwards (Fig. 5) which is detected by the counter. The counters on the electronics boards output the frequency to a computer where the data is stored and analysed.

The QCM cells are activated by spraying/dropping an antibody (AB) solution (Biosensor Applications AB, Sweden) onto the wet surface of the interface using e.g. a syringe.

The antibodies are transported via the liquid flow to the measurement QCM-cells, where they are immobilised on the crystals' pre-prepared (Biosensor Applications AB) active surfaces on the (wet) sides of the crystals. ${ }^{5}$ This increases the surface mass load of the sensor, which results in a decrease in resonance frequency on the order of 20 to $120 \mathrm{~Hz}$, (Fig. 5).

After the sensor signal stabilisation during one minute, a paper filter with narcotics traces is moved from the collector device and positioned on the interface clamping ring (Fig. 4).
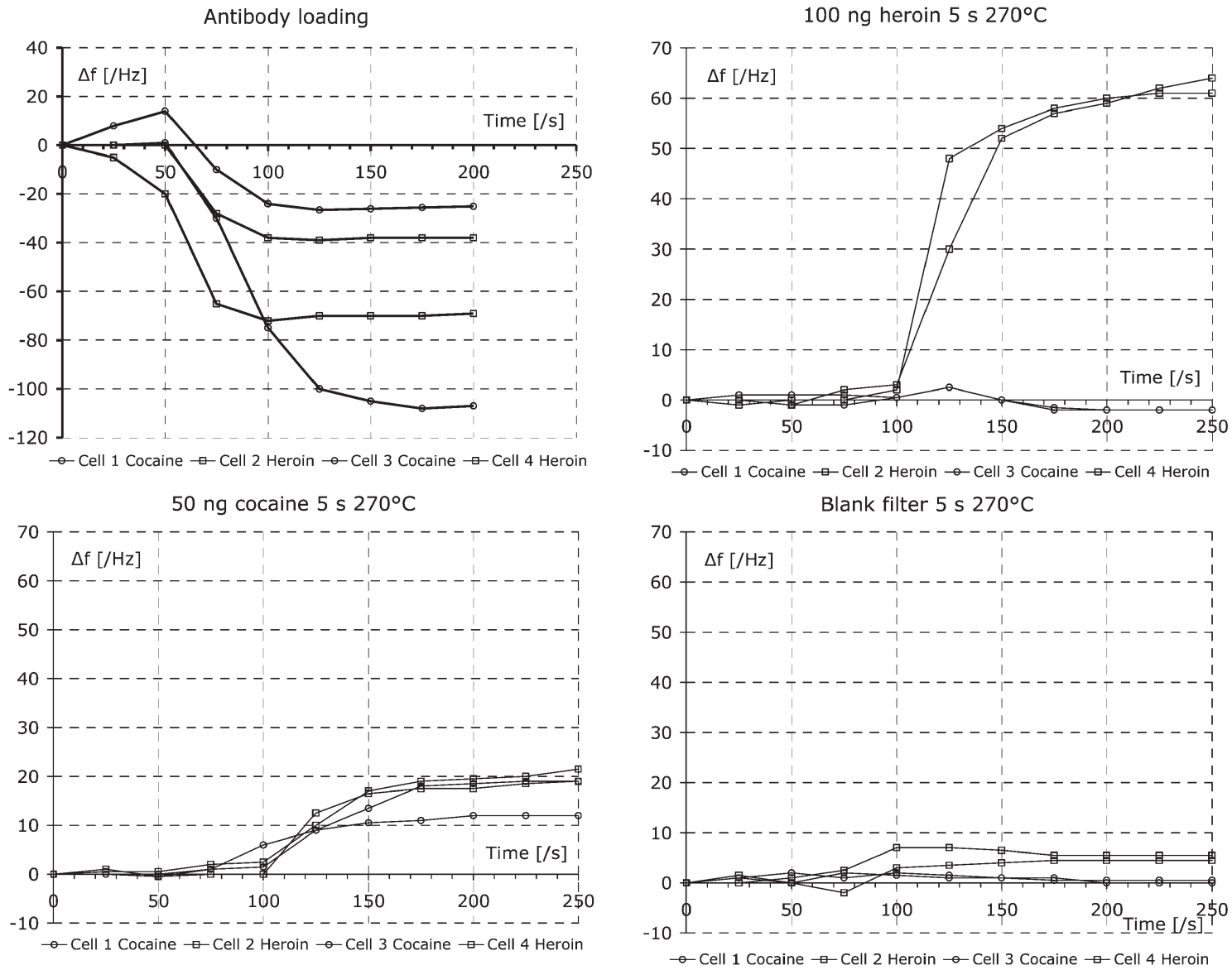

Fig. 5 Resonance frequency measurements of the heroin and cocaine sensitive measurement cells when: (top left) antibodies (mass) are dropped onto the interface, whereupon mass binds to the sensor crystal surfaces; (top right) a filter sheet containing 100 ng heroin sample is heated above the interface, whereupon only the heroin sensitive elements react; (bottom left) a filter sheet containing a 50 ng cocaine sample is heated above the interface, whereupon all sensor cells react and discrimination between cocaine and heroin sensitive sensors is not as pronounced as in heroin detection; and (bottom right) a blank sample filter is heated above the interface, where the resonance frequency shifts only slightly due to heat and salinity increase. Each heat pulse was $270{ }^{\circ} \mathrm{C}$ and lasted $5 \mathrm{~s}$. 
Table 1 Experimental—system results and discussion

\begin{tabular}{lllll}
\hline Sample & Amount & Frequency shift/Hz & Variance/Hz & Comment \\
\hline Heroin & $\sim 100 \mathrm{ng}$ & 50 & \pm 10 & Filter disk pre-prepared \\
Cocaine & $\sim 50 \mathrm{ng}$ & 15 & \pm 5 & Filter disk pre-prepared \\
Antibodies & 1 drop & -20 to -110 & \pm 30 & Drop on interface component $1^{a}$ \\
Blank & & $<10$ & \pm 3 & Clean filter disk $1^{a}$ \\
Orientation change & $90^{\circ}$ & $<1$ & $\mathrm{n} / \mathrm{a}$ & Note $2^{b}$ \\
& $180^{\circ}$ & & Note $3^{c}$
\end{tabular}

${ }^{a}$ Note 1 . This allowed a proper discrimination of signal caused by drug detection. ${ }^{b}$ Note 2 . Reference runs, performed with clean filters, gave frequency shifts of less than $10 \mathrm{~Hz}$. This frequency shift could be caused by the oven heat pulse warming of the liquid (temperature increase) and the PBS salinity change (concentration increase). See Fig. 5. ${ }^{c}$ Note 3. The gravitational invariance was tested and the system was found to be insensitive to position/orientation changes. This is particularly useful when the device is oriented upside down so that dust particles will not fall on the interface and thus contaminate or clog the device. This seemed to be the case; however, we did not test this thoroughly as we found that cleaning/rinsing of the device through a brief overflow did remove larger particles nicely.

The heater is switched on and set to reach $270{ }^{\circ} \mathrm{C}$ during $5 \mathrm{~s}$. The narcotics sample evaporates/vaporises from the filter and condenses on the PBS liquid surface of our device. The constant liquid flow (approx. $100 \mu \mathrm{min}^{-1}$ ) in the device transports the adsorbed molecules to the five sensing QCM cells downstream. The antibodies immobilised on the respective sensors are released when reached by the sample, with a resulting mass reduction and a measured increase in resonance frequency of the order of 10 to $60 \mathrm{~Hz}$ (Fig. 5). The procedure was repeated several times with heroin and cocaine-sample prepared filters (Fig. 5) as well as blank non-prepared filters.

The reaction time of the system was measured, starting the stopwatch when the heater was turned on and stopping it when the frequency shift was seen on the computer screen.

To verify that the interface functionality (priming and filling, pinch-off and dry-out, operation and robustness) does not depend on physical orientation and position, i.e. gravity does not have an influence on performance, different orientation positioning schemes were tested in the machine too. Vapour sample was introduced from underneath towards the down facing liquid surface as well as from the side towards the vertically positioned interface.

The filter-to-sensor sample transfer time was measured to be approximately $30 \mathrm{~s}$, but the system uses considerable lengths of tubing, approximately $25 \mathrm{~cm}$, due to experimental setup reasons, resulting in longer detection times (transport + dispersion) than necessary.

\section{Experimental—system results and discussion}

\section{Conclusion}

A novel, uncomplicated microfluidic interface for aerosol/ vapour-to-liquid adsorption and droplet-to-liquid transfer was designed, fabricated, characterised, integrated and tested.

Its characteristics fall within a range that is of interest for commercial applications such as sampling devices and electronic nose devices, and can be modified easily to accomplish different demands in electronic nose usage as well as lab on a chip usage or point-of-care devices.

Integration with a QCM-based electronic nose system for detection of explosives and narcotics worked well.

Detection of relevant levels of heroin and cocaine were successfully performed.

The novel system features a large "active" adsorption area (the liquid surface) as well as a low "dead" surface area (pillar tops and connector walls) in order to achieve an effective continuous flow-through system that was successfully tested with various samples in subsequent runs. Through integration and building a microfluidic flow-through system, we reduce the parasitic binding area, where sample molecules and antibodies otherwise would be consumed.

Through the miniaturisation, we obtained small liquid volumes, accommodating high sample concentration. Also gravitational invariance was achieved.

The microfluidic system design helped in decreasing detection time to accommodate rapid measurements.

The design with etched pillars and external connectors can be improved and optimised in terms of geometries and material choices for greater simplicity and greater robustness. Another potential improvement would be cooling of the liquid interface, which would be beneficial for condensation efficiency as well since it would reduce evaporation from the device.

\section{Acknowledgements}

This work was funded by VINNOVA through the SUMMIT program. We would like to thank the staff of Biosensor Applications AB for their commitment, help and support. We would also like to thank Kjell Norén at the Microsystem Technology Laboratory at the Royal Institute of Technology, Stockholm, for the help in manufacturing of the fluid connectors.

\section{References}

1 F-U. Gast and H. Fiehn, The development of integrated microfluidic systems at GeSiM, Lab Chip, 2003, 3, 6N-10N.

2 J. Melin, W. van der Wijngaart and G. Stemme, Behaviour and Design Considerations for Continuous Flow Closed-Open-Closed Liquid Microchannels, Lab Chip, 2005, 5(6), 682.

3 P. Månsson and D. Rönnholm, Biosensor Technology-an Introduction, Biosensor Applications, Sundbyberg, 2005. Available from: http://www.biosensor.se.

4 P. Månsson, H. Andersson, J. Smith, K. Jensen and T. Aastrup, System, Device and Method for Detection of Several Individual Analytes in a Solution, and a Disposable Flow Cell for Use Therein, Patent application, PCT/SE2003/001038, 2003.

5 P. Månsson and B. Liedberg, Coated Metal Surface on Solid Support for Displacement Reactions, Patent application, PCT/2003/ 001037, 2003.

6 P. Månsson, J. Smith and A.-C. Hellgren, Transfer of a Sample from a Solid Support into a Liquid, Patent application, PCT/SE02/ 02098, 2003.

7 F. M. White, Fluid Mechanics, McGraw-Hill, New York, 1979. 